\title{
Regimes hídricos e doses de fósforo em cafeeiro
}

\author{
Ana Carolina Mera (1); Carlos Alberto da Silva Oliveira ( $\left.{ }^{*}\right)$; Antônio Fernando Guerra ( ${ }^{2}$ ); \\ Gustavo Costa Rodrigues ( $\left.{ }^{2}\right)$
}

(') Universidade de Brasília, Faculdade de Agronomia e Medicina Veterinária, Brasília (DF).

(2) Embrapa Cerrados, Caixa Postal 08223, 73301-970 Planaltina (DF).

(*) Autor correspondente: dasilvao@unb.br

Recebido: 5/mai./2009; Aceito: 14/set./2010.

\section{Resumo}

O trabalho objetivou estudar os crescimentos vegetativo e reprodutivo e a produtividade de cafeeiro sob três regimes hídricos e quatro doses de adubação fosfatada, no segundo ano após a poda, em solo de Cerrado. A cultivar utilizada foi a Catuaí Rubi MG 1192, Coffea arabica L., com 7.143 plantas ha-1. Os regimes hídricos aplicados foram: sem suspensão da irrigação, feita o ano todo (I); e a partir de 24/6/07, com suspensão da irrigação por 70 dias ( $\mathrm{SI}_{70}$ ); e com suspensão da irrigação por 109 dias $\left(\mathrm{SI}_{109}\right)$. Os tratamentos $\mathrm{SI}_{70}$ e $\mathrm{SI}_{109}$ foram interrompidos por uma chuva de $12 \mathrm{~mm}$ (1.\%10/07), que visualmente induziu a floração. As quatro doses anuais de adubação fosfatada foram: $0\left(P_{0}\right), 100\left(P_{100}\right), 200\left(P_{200}\right)$ e $400\left(P_{400}\right)$ kg de $P_{2} O_{5}$ ha-1. $\mathrm{O}$ delineamento experimental foi o de blocos completos ao acaso com o regime hídrico na parcela e a dose de fósforo na subparcela, com três repetições. As doses de fósforo $\left(\mathrm{P}_{200}\right)$ e $\left(\mathrm{P}_{400}\right)$ proporcionaram aumento no comprimento e área foliar de ramos, número de frutos e na produtividade de grãos. Os regimes hídricos $\mathrm{SI}_{70}$ e $\mathrm{SI}_{109}$ proporcionaram as maiores porcentagens de frutos cereja e a maior e menor produtividade de grãos respectivamente.

Palavras-chave: crescimento, déficit hídrico, irrigação, nutrição, produtividade.

\section{Water regimes and phosphorus levels on coffee plants}

\section{Abstract}

This work aimed to study the vegetative and reproductive growth and yield of coffee plants under three water regimes and four phosphorus fertilization doses, on second year after pruning on cerrado soil. The cultivar used was Catuai Rubi, MG 1192, Coffea arabica L. with 7,143 plants ha ${ }^{-1}$. Water regimes were: all year round irrigation (I); after June $24^{\text {th }}$, 2007, irrigation was stopped for 70 days $\left(\mathrm{SI}_{70}\right)$ and for 109 days $\left(\mathrm{SI}_{109}\right)$. Treatments $\mathrm{SI}_{70}$ e $\mathrm{SI}_{109}$ were interrupted with a rainfall of $12 \mathrm{~mm}($ October $\left.1^{\text {st }}, 2007\right)$, which visually caused flowering initiation. The four phosphorus doses were: $0\left(P_{0}\right), 100\left(P_{100}\right), 200\left(P_{200}\right)$ e $400\left(P_{400}\right)$ $\mathrm{kg}$ of $\mathrm{P}_{2} \mathrm{O}_{5} \mathrm{ha}^{-1}$. The experiment was arranged in randomized complete block design for water regime with phosphorus doses as split plot and three replications. The highest doses of phosphorus $\left(P_{200}\right)$ and $\left(P_{400}\right)$ provided increases in branch length, leaf area, number of fruits and grain yield. Water regimes $\mathrm{SI}_{70}$ and $\mathrm{SI}_{109}$ provided higher cherry fruit percentage and the highest and lowest grain yield, respectively.

Key words: irrigation, growth, nutrition, water deficit, yield.

\section{INTRODUÇÃO}

Em 2008, o Brasil produziu 46 milhóes de sacas de 60 $\mathrm{kg}$ de café beneficiado, segunda maior safra dos últimos dez anos, e para 2009, o país deverá colher entre 36,9 e 38,8 milhóes de sacas das quais aproximadamente $70 \%$ são de café arábico (Conab, 2009).

Com o crescente avanço da cafeicultura de cerrado, devido às restriçóes hídricas e à baixa fertilidade natural desses solos, torna-se necessária a aplicação de técnicas adequadas de irrigação e adubação.

A periodicidade de crescimento do cafeeiro está associada a diversos fatores ambientais, entre eles o suprimento de água e nutrientes (Damatta e Rena, 2002). Por exemplo, a ocorrência do déficit hídrico em certas fases do ciclo fenológico, como a que antecede no início da floração, pode comprometer a produtividade do cafeeiro (Silva et al., 2009).

Existem diversas pesquisas sobre a necessidade do uso de um déficit hídrico controlado visando à indução floral uniforme e à consequente qualidade final dos grãos obtendo-se maior rentabilidade (CARR, 2001; Crisoto et al., 1992; Drinnan e Menzel, 1994).

SiLva et al. (2009) observaram que a suspensão da irrigaçáo no período de inverno melhorou a uniformidade no desenvolvimento dos frutos do cafeeiro arábica. Nascimento (2008) observou que a irrigação durante o ano todo reduziu a emissão de flores e os tratamentos com paralisação da irrigação tiveram resultados superiores aos tratamentos sem irrigação e 
irrigação durante todo o ano para a maioria das variáveis avaliadas.

Em relação à adubação fosfatada, não deve ser fundamentada exclusivamente na safra de grãos em formação, pois as aplicaçôes de fertilizantes também devem ter por objetivo o crescimento de novos ramos e nós para a safra futura (Guerra et al., 2006). Assim, visando reduzir a bienalidade da produção do cafeeiro, estes autores indicam a dose de $300 \mathrm{~kg}$ de $\mathrm{P}_{2} \mathrm{O}_{5} \mathrm{ha}^{-1}$ ano $^{-1}$ para: colher anualmente de 3600 a $4200 \mathrm{~kg} \mathrm{ha}^{-1}$; atender a necessidade de $\mathrm{P}$ para o enchimento de grãos; manter o crescimento dos ramos vegetativos e reprodutivos; e formar e diferenciar as gemas para a safra futura.

Estudando o crescimento inicial da cultivar Rubi MG 1192 irrigada, NAZARENo et al. (2003) concluíram que o $\mathrm{N}$ e K afetaram o número de ramos plagiotrópicos e o número de nós com gemas por planta, mas o $\mathrm{P}$ não afetou. Os autores mencionam que, provavelmente, o $\mathrm{P}$ usado na adubaçáo de correção antes do plantio das mudas, e na cova de plantio, foi suficiente para suprir a necessidade do cafeeiro em formação. Todavia, dados de produção afetados por este nutriente estão pouco disponíveis na literatura (Damatta et al., 2007).

Este trabalho objetivou estudar os crescimentos vegetativo e reprodutivo e a produtividade do cafeeiro submetido a três regimes hídricos e quatro doses de adubação fosfatada, no segundo ano após a poda em plantas adensadas, em solo de Cerrado.

\section{MATERIAL E MÉTODOS}

O trabalho foi realizado em Planaltina, DF (latitude de 15³4'30"S e longitude 47042'30"W, com altitude média de 1.000 m). Segundo a classificação de Köppen, o clima é do tipo Aw, tropical chuvoso de inverno seco, com deficiência hídrica em torno de $168 \mathrm{~mm}$. Dados de temperatura do ar e precipitaçáo pluvial observados ao impor os regimes hídricos estão disponíveis na figura 1 . O solo da

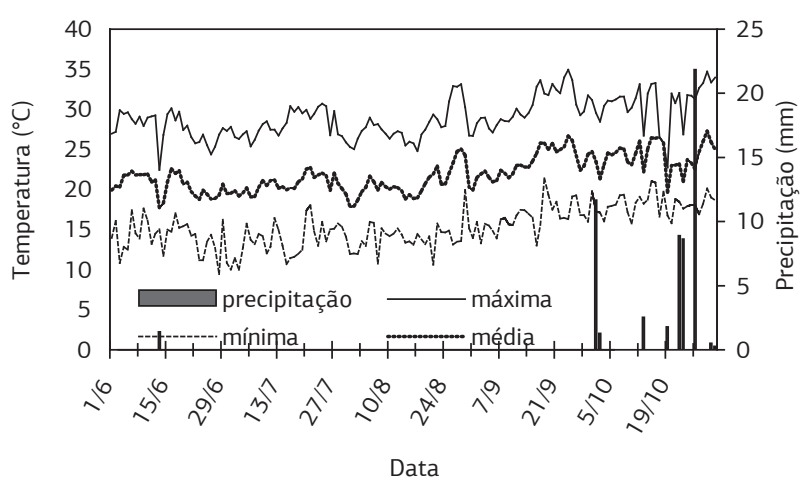

Figura 1. Dados diários de temperatura máxima, mínima e média do ar e precipitaçáo pluvial no período de $1 . \%$ a 31/10/2007. No mês 10 ocorreu o total de $57,4 \mathrm{~mm}$ de precipitação pluvial. área experimental foi classificado como Latossolo Vermelho-Escuro distrófico, de textura argilosa.

A cultivar utilizada foi a Catuaí Rubi, linhagem MG 1192 (Coffea arabica L). O espaçamento foi de 2,80 m entre linhas x $0,50 \mathrm{~m}$ entre plantas, com área útil por planta de $1,4 \mathrm{~m}^{2}$ e população de 7.143 plantas ha ${ }^{-1}$, caracterizando plantio adensado.

O delineamento experimental foi o de blocos completos ao acaso com o regime hídrico na parcela e a dose de $\mathrm{P}_{2} \mathrm{O}_{5}$ ha $^{-1}$ na subparcela, com três repetiçóes.

$\mathrm{O}$ experimento foi desenvolvido em uma área irrigada por pivô central submetida a três regimes hídricos: sem suspensão da irrigação, feita o ano todo (I); a partir de 24/6/07, com suspensão da irrigação por 70 dias $\left(\mathrm{SI}_{70}\right)$, até $2 / 9$; e com suspensão da irrigação por 109 dias $\left(\mathrm{SI}_{109}\right)$, até 11/10, quando a chuva de $12 \mathrm{~mm}(01 / 10)$ e as temperaturas mínimas mais elevadas, visualmente, induziram a floração. A irrigação continuou após os tratamentos. A área irrigada foi dividida em setores de 2 ha para cada regime hídrico.

O manejo da irrigação por pivô central foi feito usando medidas do conteúdo de água no solo pela leitura do sensor Theta Probe tipo ML2X instalado na linha de plantio nas profundidades de: 10, 20, 30, 50 e $100 \mathrm{~cm}$. Foram feitas leituras diárias do conteúdo de água nas diferentes profundidades em todos os regimes hídricos. Foram feitas aplicaçóes de água até a condiçâo de capacidade de campo sempre que a leitura do sensor instalado a $10 \mathrm{~cm}$ de profundidade acusasse valores em torno de $0,29 \mathrm{~cm}^{3} \mathrm{~cm}^{-3}$, ou seja, sempre que as plantas consumissem aproximadamente $50 \%$ da água disponível no perfil de solo de $40 \mathrm{~cm}$.

Os tratamentos de $\mathrm{P}_{2} \mathrm{O}_{5}$ foram: 0, 100, 200 e 400 $\mathrm{kg} \mathrm{ha}^{-1} \mathrm{ano}^{-1}$. A fonte utilizada foi o superfosfato triplo e a aplicação realizada em cobertura, em faixa e na projeção da copa das plantas. Foi parcelada em duas aplicações, sendo $2 / 3$ da dose $\left(0,66,7,133,3\right.$ e 266,7 $\left.\mathrm{kg} \mathrm{ha}^{-1}\right)$ aplicada em setembro, antes do retorno das irrigaçóes, e $1 / 3$ da dose $\left(0,33,3,66,7\right.$ e 133,3 $\left.\mathrm{kg} \mathrm{ha}^{-1}\right)$ no fim de dezembro.

Em todos os tratamentos foram aplicados anualmente $500 \mathrm{~kg} \mathrm{ha}^{-1}$ de nitrogênio (uréia), $500 \mathrm{~kg} \mathrm{ha}^{-1} \mathrm{de} \mathrm{K}_{2} \mathrm{O}$ e $100 \mathrm{~kg} \mathrm{ha}^{-1}$ de FTE BR10 $(\mathrm{Zn}=7 \% ; \mathrm{B}=2,5 \% \mathrm{Cu}=1 \%$; $\mathrm{Fe}=4 \% ; \mathrm{Mn}=4 \% ; \mathrm{Mo}=0,1 \% ; \mathrm{Co}=0,1 \%)$. A adubação com $\mathrm{N}$ e $\mathrm{K}$ foi dividida em quatro parcelamentos, com início em setembro/2007 e término em fevereiro/2008.

Segundo método adotado pela Embrapa (1997), as características químicas do solo nos diversos tratamentos de P, em março de 2007, estão relacionadas na Tabela 1.

Em setembro/outubro de 2005 todas as plantas da área passaram por poda do tipo decote a 1,60 $\mathrm{m}$ de altura e esqueletamento de $0,2 \mathrm{~m}$ na parte superior e $0,4 \mathrm{~m}$ na parte inferior. As medidas de crescimento vegetativo e reprodutivo dos ramos foram realizadas acima do decote, conforme se segue.

No ramo ortotrópico foram avaliados: crescimento em altura; número de nós; e de ramos primários, durante 165 dias (2/11/2007 a 15/4/2008), em amostras de três 
plantas por tratamento, nos três regimes hídricos e nas doses de $\mathrm{P}_{2} \mathrm{O}_{5}: 0,200$ e $400 \mathrm{~kg} \mathrm{ha}^{-1}$.

Nos ramos plagiotrópicos primários de todos os tratamentos foram avaliados (maio de 2008): $\mathrm{r}_{1}$, ramo novo com crescimento vegetativo e que ainda não produziu; $r_{2}$, ramo com crescimento vegetativo e reprodutivo (frutos) em $1^{\text {a }}$ produçáo; e $\mathrm{r}_{3}$, ramo com crescimento vegetativo, ramificações secundárias e terciárias e crescimento reprodutivo (frutos) em $2^{a}$ produçáo. Foram coletados quatro ramos de cada tipo por planta em cada tratamento.

Nos ramos $\mathrm{r}_{1} ; \mathrm{r}_{2}$; e $\mathrm{r}_{3}$ foram avaliados: $\mathrm{r}_{1}$ - número de novo nó sem frutos e de folhas; diâmetro da base, comprimento e área foliar do ramo; $\mathrm{r}_{2}$ - número de novo nó sem frutos (diferença do número total de nó e o número deles até o último nó com frutos); comprimento e área foliar do ramo; número de nó com frutos, de frutos e de frutos por nó no ramo; e $\mathrm{r}_{3}$ - número de novo nó sem frutos e de ramificaçáo secundária; comprimento e área foliar do ramo; e número de nó com frutos e de frutos.

De acordo com o estágio de maturação os frutos foram classificados em: verde, verde cana, cereja e seco ao ar.

A produtividade total foi obtida de seis plantas úteis por parcela. Após a colheita os frutos foram secos, até atingirem $12 \%$ de umidade, e posteriormente beneficiados.

As duas variáveis independentes estudadas combinaram simultaneamente um fator assumido como qualitativo e não estruturado (regime hídrico/climático/temporal de suspen- são da irrigação) e um fator quantitativo (doses de $\mathrm{P}_{2} \mathrm{O}_{5}$ ). Assim, em um primeiro momento, os dados obtidos nos ramos ortotrópicos e plagiotrópicos foram submetidos à análise de variância e as médias comparadas por teste estatístico de Duncan a 5\% de probabilidade, possibilitando verificar possíveis interaçōes. A relação entre duas variáveis de uma mesma população foi estabelecida através do coeficiente de correlação de Pearson (r). Em um segundo momento, foi feita a análise de regressão em função das doses de fósforo. $\mathrm{O}$ aplicativo MSTATC foi utilizado para as análises.

\section{RESULTADOS E DISCUSSÃO}

\section{Ramo ortotrópico}

O crescimento em altura $(16,0)$, o número de nó $(6,8)$ e de ramos primários $(11,1)$ do cafeeiro, obtidos acima de $1,60 \mathrm{~m}$ de altura, foram significativamente maiores no tratamento $\mathrm{SI}_{109}$, entretanto, não foram afetados pelas doses de $\mathrm{P}_{2} \mathrm{O}_{5}$ (Tabela 2). Por outro lado foram significativamente menores no tratamento $\mathrm{SI}_{70}$. Resultados semelhantes foram encontrados por GuERRA et al. (2006).

A suspensão da irrigação por um período longo (> 30 dias, por exemplo) pode causar um déficit hídrico no cafeeiro $\left(\mathrm{SI}_{70}\right)$, e provocar aumento na uniformidade de floração e na safra em formação. Todavia, a hipótese do

Tabela 1. Características químicas do solo (Embrapa, 1997), em 03/2007, na profundidade de 0 a $20 \mathrm{~cm}$ e nos tratamentos de $\mathrm{P}_{2} \mathrm{O}_{5}: 0$, 100,200 e $400 \mathrm{~kg} \mathrm{ha}^{-1}$ ano $^{-1}$

\begin{tabular}{|c|c|c|c|c|c|c|c|c|c|c|c|c|c|c|}
\hline \multirow{2}{*}{ Trat. } & \multirow{2}{*}{$\mathrm{pH}\left(\mathrm{H}_{2} \mathrm{O}\right)$} & \multirow{2}{*}{$\mathrm{pH}\left(\mathrm{CaCl}_{2}\right)$} & $\mathrm{Al}^{3+}$ & $\mathrm{Ca}^{2+}$ & $\mathrm{Mg}$ & $\mathrm{H}+\mathrm{Al}^{3+}$ & $\mathbf{P}$ & K & $\mathrm{Cu}$ & $\mathrm{Fe}$ & Mn & $\mathrm{Zn}$ & \multirow{2}{*}{$\begin{array}{c}\text { M.O. } \\
\text { dag } \text { kg }^{-1}\end{array}$} & \multirow{2}{*}{ Sat.Al } \\
\hline & & & \multicolumn{4}{|c|}{$\mathrm{cmol}_{\mathrm{c}} \mathrm{dm}^{-3}$} & \multicolumn{6}{|c|}{$\mathrm{mg} \mathrm{dm}^{-3}$} & & \\
\hline $\mathrm{P}_{0}$ & 5,0 & 4,2 & 1,1 & 0,7 & 0,2 & 6,5 & 2,9 & 117,2 & 0,9 & 32,3 & 4,4 & 3,6 & 3,4 & 19,3 \\
\hline$P_{100}$ & 5,1 & 4,2 & 0,7 & 1,1 & 0,2 & 6,5 & 11,1 & 124,4 & 0,4 & 30,0 & 4,2 & 2,9 & 3,4 & 20,4 \\
\hline$P_{200}$ & 5,1 & 4,1 & 0,9 & 0,8 & 0,2 & 7,1 & 20,1 & 154,4 & 0,4 & 33,1 & 4,5 & 2,1 & 2,7 & 22,8 \\
\hline $\mathrm{P}_{400}$ & 5,1 & 4,1 & 0,6 & 1,0 & 0,3 & 6,9 & 31,0 & 154,4 & 0,5 & 30,3 & 3,9 & 2,4 & 3,0 & 23,6 \\
\hline
\end{tabular}

Tabela 2. Ramo ortotrópico. Crescimento em altura, número de nó e de ramos primários do cafeeiro Rubi MG 1192, obtidos acima de $1,60 \mathrm{~m}$ da altura da poda e durante 165 dias (2/11/2007 a 15/4/2008), em função de três regimes hídricos: sem suspensão da irrigação, feita o ano todo (I); e a partir de 24/6/07, com suspensão da irrigação por 70 dias $\left(\mathrm{SI}_{70}\right)$, até 2/09; e com suspensão da irrigação por 109 dias $\left(\mathrm{SI}_{109}\right)$, até $11 / 10$ e quatro doses de $\mathrm{P}_{2} \mathrm{O}_{5}: 0\left(\mathrm{P}_{0}\right), 200\left(\mathrm{P}_{200}\right)$ e $400\left(\mathrm{P}_{400}\right) \mathrm{kg} \mathrm{ha}^{-1}$ ano ${ }^{-1}$

\begin{tabular}{|c|c|c|c|c|c|}
\hline \multirow{2}{*}{ Variável } & \multirow{2}{*}{ Regime Hídrico } & \multicolumn{4}{|c|}{ Dose de $\mathrm{P}_{2} \mathrm{O}_{5} \mathrm{~kg} \mathrm{ha}^{-1}$ ano $^{-1}$} \\
\hline & & $P_{0}$ & $\mathbf{P}_{200}$ & $P_{400}$ & Médias \\
\hline \multirow{4}{*}{ Crescimento em altura $(\mathrm{cm})$} & I & 12,2 & 15,7 & 11,2 & $12,1 \mathrm{~b}$ \\
\hline & $\mathrm{SI}_{70}$ & 9,7 & 12,7 & 12,2 & $11,6 b$ \\
\hline & $\mathrm{SI}_{109}$ & 11,7 & 17,7 & 18,7 & $16,0 \mathrm{a}$ \\
\hline & Médias & 11,2 & 15,3 & 14,1 & \\
\hline \multirow{4}{*}{ Número de nó } & I & 5,4 & 5,5 & 6,1 & $5,7 \mathrm{~b}$ \\
\hline & $\mathrm{SI}_{70}$ & 4,7 & 5,2 & 5,5 & $5,2 \mathrm{c}$ \\
\hline & $\mathrm{SI}_{109}$ & 6,4 & 6,8 & 7,1 & $6,8 \mathrm{a}$ \\
\hline & Médias & 5,5 & 5,8 & 6,2 & \\
\hline \multirow{4}{*}{ Número de ramos primários } & 1 & 8,8 & 8,8 & 9,6 & $9,0 \mathrm{~b}$ \\
\hline & $\mathrm{SI}_{70}$ & 7,3 & 8,8 & 9,0 & $8,4 \mathrm{c}$ \\
\hline & $\mathrm{SI}_{109}$ & 9,8 & 11,8 & 11,9 & $11,1 \mathrm{a}$ \\
\hline & Médias & 8,6 & 9,8 & 10,2 & \\
\hline
\end{tabular}

Médias seguidas pela mesma letra na coluna não diferem entre si ao nível de probabilidade de $5 \%$. 
déficit hídrico excessivamente prolongado $\left(\mathrm{SI}_{109}\right)$ reduzir a safra em formaçáo ou crescimento reprodutivo e favorecer o crescimento vegetativo, também tem fundamento, e se sustenta diante dos resultados observados.

Ainda, com irrigação durante o ano todo e com suspensão da irrigação por 70 dias, o crescimento similar em altura da planta e o número de nós diferenciado podem ser atribuídos ao alongamento dos entrenós. Este fato sugere que os fotoassimilados são deslocados de forma diferente para as diversas partes da planta, em funçáo do regime hídrico.

As doses menores de $\mathrm{P}_{2} \mathrm{O}_{5}$ foram suficientes para atender a demanda deste tipo de crescimento do cafeeiro. A ausência de resposta ao $P$ neste crescimento também foi observada por Nazareno et al. (2003). Segundo MaTIELlo et al. (2005), no período de rápido crescimento vegetativo e reprodutivo das plantas, de outubro a abril, também ocorrem mais de $80 \%$ do consumo de nutrientes que são destinados à frutificação da safra pendente. Assim, havendo reduçáo da safra pendente, os fotoassimilados podem ser redirecionados para as partes vegetativas, aumentando este crescimento. Tal crescimento não tem efeito direto na safra em formação, mas, certamente, na safra futura. Esta hipótese se fortalece diante do efeito do $\mathrm{P}$ observado nos ramos plagiotrópicos, principalmente ramos $\mathrm{r}_{3}$, responsáveis pela produtividade de grãos, conforme se segue.

\section{Ramos plagiotrópicos tipo $r_{1}$}

Nestes ramos houve interação entre regime hídrico e doses de fósforo para número total de nós e de folhas (Tabela 3). Tal não ocorreu para o diâmetro da base, comprimento do ramo e área foliar.

Exceto o número de nós, todos os outros quatro tipos de crescimento vegetativo tiveram maiores valores $(25,5$; 4,9; 37,4; e 745,9) no déficit hídrico $\mathrm{SI}_{109}$, mais prolongado. Este fato é nitidamente evidenciado através dos respectivos interceptos das equaçóes de regressóes ajustadas para cada regime hídrico. Analogamente ao crescimento do ramo ortotrópico, o déficit hídrico prolongado proporcionou maiores valores de crescimento neste tipo de ramo plagiotrópico, com menor impacto sobre a safra em

Tabela 3. Ramo plagiotrópico $\mathrm{r}_{1}$ com folhas e que ainda não produziu. Número de novo nó sem frutos e de folhas, e diâmetro (mm), comprimento $(\mathrm{cm})$ e área foliar $\left(\mathrm{cm}^{2}\right)$ do ramo obtidos acima de 1,60 m do cafeeiro Rubi MG 1192, em maio/2008, em funçáo de três regimes hídricos $(\mathrm{RH})$ : irrigação o ano todo $(\mathrm{I})$; suspensão da irrigação por 70 dias $\left(\mathrm{SI}_{70}\right)$, a partir de 24 de junho; e por 109 dias $\left(\mathrm{SI}_{109}\right)$, quando a chuva induziu a floração e de quatro doses de $\mathrm{P}_{2} \mathrm{O}_{5}: 0\left(\mathrm{P}_{0}\right), 100\left(\mathrm{P}_{100}\right), 200\left(\mathrm{P}_{200}\right)$ e $400\left(\mathrm{P}_{400}\right) \mathrm{kg} \mathrm{ha}^{-1}$ ano ${ }^{-1}$; respectivas equaçóes de regressão, $y=f(x)$, sendo $x$ a dose de fósforo, $R^{2}$ e significância

\begin{tabular}{|c|c|c|c|c|c|c|c|c|}
\hline RH & $\mathbf{P}_{0}$ & $\mathbf{P}_{100}$ & $\mathbf{P}_{200}$ & $\mathbf{P}_{400}$ & Méd. & $y=f(x)$ & $\mathbf{R}^{2}$ & \\
\hline \multicolumn{9}{|c|}{ Número de novo nó sem frutos } \\
\hline I & $10,7 \mathrm{f}$ & $13,1 \mathrm{de}$ & 14,9 abc & $15,1 \mathrm{ab}$ & 13,4 & $y=-5 \cdot 10^{-5} x^{2}+0,034 x+10,7$ & 0,99 & * \\
\hline $\mathrm{SI}_{70}$ & $12,4 \mathrm{e}$ & $12,5 \mathrm{e}$ & 13,9 bcd & 15,6 a & 13,6 & $y=0,0086 x+12,1$ & 0,95 & * \\
\hline $\mathrm{SI}_{109}$ & $12,0 \mathrm{e}$ & $12,1 \mathrm{e}$ & $13,8 \mathrm{~cd}$ & $13,8 \mathrm{~cd}$ & 12,9 & $y=-1 \cdot 10^{-5} x^{2}+0,010 x+11,8$ & 0,80 & NS \\
\hline Méd. & 11,7 & 12,5 & 14,2 & 14,8 & & & & \\
\hline \multicolumn{9}{|c|}{ Número de folhas } \\
\hline I & $11,4 \mathrm{~d}$ & 20,0 bc & $22,7 \mathrm{abc}$ & 22,8 abc & 19,3 & $y=-2 \cdot 10^{-4} x^{2}+0,089 x+11,7$ & 0,98 & NS \\
\hline $\mathrm{SI}_{70}$ & $19,0 \mathrm{c}$ & 20,1 bc & $24,8 \mathrm{ab}$ & $25,0 \mathrm{ab}$ & 22,3 & $y=-5.10^{-5} x^{2}+0,036 x+18,4$ & 0,88 & NS \\
\hline $\mathrm{SI}_{109}$ & $21,0 \mathrm{abc}$ & $21,7 \mathrm{abc}$ & $24,8 \mathrm{ab}$ & $25,5 \mathrm{a}$ & 23,3 & $y=-2 \cdot 10^{-5} x^{2}+0,022 x+20,6$ & 0,90 & NS \\
\hline Méd. & 17,1 & 20,6 & 24,2 & 24,4 & & & & \\
\hline \multicolumn{9}{|c|}{ Diâmetro da base do ramo } \\
\hline I & 4,1 & 4,1 & 4,8 & 4,9 & $4,3 \mathrm{C}$ & $y=-1 \cdot 10^{-6} x^{2}+0,004 x+4,0$ & 0,81 & NS \\
\hline $\mathrm{SI}_{70}$ & 4,1 & 4,1 & 4,1 & 4,7 & $4,5 \mathrm{~B}$ & $y=7.10^{-6} x^{2}-0,0013 x+4,1$ & 0,99 & NS \\
\hline $\mathrm{SI}_{109}$ & 4,7 & 4,7 & 5,0 & 5,1 & $4,9 \mathrm{~A}$ & $y=-9 \cdot 10^{-7} x^{2}+0,0015 x+4,7$ & 0,86 & NS \\
\hline Méd. & $4,3 \mathrm{c}$ & $4,3 \mathrm{c}$ & $4,6 \mathrm{~b}$ & $4,9 \mathrm{a}$ & & $y=1.10^{-6} x^{2}+0,001 x+4,3$ & 0,94 & * \\
\hline \multicolumn{9}{|c|}{ Comprimento } \\
\hline I & 29,0 & 35,2 & 35,4 & 35,9 & $33,9 \mathrm{C}$ & $y=-9 \cdot 10^{-5} x^{2}+0,053 x+29,5$ & 0,91 & NS \\
\hline $\mathrm{SI}_{70}$ & 32,3 & 33,7 & 36,1 & 40,4 & $35,6 \mathrm{~B}$ & $y=1.10^{-5} x^{2}+0,0165 x+32,2$ & 0,99 & ** \\
\hline $\mathrm{SI}_{109}$ & 34,1 & 35,3 & 38,8 & 41,2 & $37,4 \mathrm{~A}$ & $y=0,0186 x+34,1$ & 0,95 & * \\
\hline Méd. & $31,8 \mathrm{c}$ & $34,7 \mathrm{~b}$ & $36,7 \mathrm{~b}$ & 39,2 a & & $y=-3.10^{-5} x^{2}+0,031 x+31,8$ & 1,00 & * \\
\hline \multicolumn{9}{|c|}{ Área Foliar } \\
\hline I & 264 & 558 & 504 & 638 & $491 C$ & $y=-0,0028 x^{2}+1,936 x+299$ & 0,81 & NS \\
\hline $\mathrm{SI}_{70}$ & 549 & 531 & 693 & 732 & $626 \mathrm{~B}$ & $y=-0,0004 x^{2}+0,678 x+527$ & 0,80 & NS \\
\hline $\mathrm{SI}_{109}$ & 599 & 659 & 827 & 897 & $745 \mathrm{~A}$ & $y=0,7801 x+609,38$ & 0,91 & * \\
\hline Méd. & $471 \mathrm{c}$ & $583 b$ & $675 \mathrm{ab}$ & 756 a & & $y=-0,0015 x^{2}+1,311 x+470$ & 1,00 & * \\
\hline
\end{tabular}

Médias seguidas pela mesma letra minúscula na linha e maiúscula na coluna não diferem entre si ao nível de probabilidade de $5 \%$. ****: Significativo a $5 \%$ e $1 \%$ de probabilidade respectivamente. NS: nấo significativo a $5 \%$ de probabilidade. 
formaçáo e maior impacto sobre a safra futura. Este efeito é coerente com menor partição de fotoassimilados observada em um primeiro momento para as reduzidas partes reprodutivas e, em um segundo momento, maior partição para partes vegetativas aumentadas, ou seja, diante da baixa demanda por parte dos chumbinhos/grãos os fotoassimilados são redirecionados para as folhas e ramos. Assim, a hipótese do déficit hídrico excessivamente prolongado $\left(\mathrm{SI}_{109}\right)$ reduzir a safra em formação ou crescimento reprodutivo e favorecer o crescimento vegetativo, é fortalecida diante dos resultados observados.

Diferentemente do ramo ortotrópico, o aumento das doses de $\mathrm{P}_{2} \mathrm{O}_{5}$ levou a um aumento nos valores $(15,6$; 25,$5 ; 4,9 ; 39,2$; e 756,1) das cinco variáveis de crescimento analisadas no ramo $r_{1}$, dentro dos três regimes hídricos. A maior quantidade de fósforo na soluçáo do solo ajuda a explicar este resultado. Todavia, em termos de diâmetro, comprimento e área foliar os efeitos do déficit hídrico de 70 e 109 dias, reduziram $(4,5 ; 35,6 ; 37,4)$ e aumentaram $(4,9 ; 37,4$; e 645,9), respectivamente, os efeitos das doses de fósforo. O número total de nós foi a única variável de crescimento a ser reduzida $(13,8)$ diante do déficit hídrico de 109 dias, provavelmente, por variar inversamente com o comprimento do ramo $r_{1}$.

Os valores negativos dos coeficientes do termo quadrático das equações de regressões ajustadas sugerem que as doses de fósforo utilizadas fornecem funçôes côncavas para baixo que podem atingir ou não um valor máximo no intervalo de doses estudado. Por outro lado, os valores positivos sugerem funções convexas que não atingiram um valor máximo de crescimento vegetativo no intervalo das doses estudadas.

No déficit hídrico de 109 dias, o número de folhas não diferiu significativamente em função das doses de fósforo, ocorrendo os maiores valores $(21,0 ; 21,7 ; 24,8$; $25,5)$. Na irrigaçáo durante o ano todo houve perda de $46,7 \%$ de folhas, enquanto na suspensão da irrigação por 70 e 109 dias,a perda foliar foi de $23,4 \%$ e $12,5 \%$, respectivamente. O déficit hídrico, ao contrário da irrigação o ano todo pode proporcionar microclima favorável à redução de patógenos, como a ferrugem e outros, reduzindo a queda de folhas e favorecendo o crescimento vegetativo. Assim, as doses mais elevadas de fósforo, além de reduzir a queda, podem auxiliar na retenção e no fortalecimento das folhas, tornando a planta mais resistente a doenças.

O diâmetro da base, o comprimento do ramo e a área foliar tiveram as mesmas diferenças significativas nas médias dos regimes hídricos e elevados coeficientes de determinação $(0,95 ; 1,00$; e 1,00$)$ das equações de regressão ajustados em função das doses de fósforo. Conclui-se que, pelo menos, $95 \%$ das variações das funções $y=f(x)$ podem ser "explicadas" pelas doses de fósforo, ficando $5 \%$ sem explicação. Em plantas irrigadas o ano todo as médias foram menores $(4,3 ; 33,9$; e 491,5$)$, enquanto em plantas submetidas à suspensão da irrigação por 109 dias observaram-se as maiores $(4,9 ; 37,4$; e 745,9$)$.

O número de folhas e a área foliar ajudam a explicar o crescimento vegetativo em diâmetro da base e comprimento do ramo juntamente com a dose de $\mathrm{P}_{2} \mathrm{O}_{5}$. O maior número de folhas associado ao aumento de área foliar em função de fósforo pode indicar um aumento da superfície fotossintética e de fotoassimilados, explicando assim, o aumento do diâmetro da base e do comprimento do ramo com o aumento das doses de $\mathrm{P}_{2} \mathrm{O}_{5}$.

\section{Ramos plagiotrópicos tipo $\mathbf{r}_{2}$}

Provavelmente surgido após o retorno da irrigação em setembro/2006, este ramo de $1^{\text {a }}$ colheita floresceu em setembro/2007 e produziu parte da safra de maio/2008 quando foi analisado. Só houve dupla interação para número de novos nós neste tipo de ramo (Tabela 4). As demais variáveis vegetativas e reprodutivas não interagiram.

Quando a irrigação foi suspensa por 70 dias, o número de novo nó sem frutos das doses 0,100 e $200 \mathrm{~kg}$ de $\mathrm{P}_{2} \mathrm{O}_{5}$ ha $^{-1}$ não diferiu significativamente entre si $(10,6$; 10,6; e 12,5), mas diferiu dos regimes hídricos I e $\mathrm{SI}_{109}$, que não foram afetados pelas doses de $\mathrm{P}_{2} \mathrm{O}_{5}$ aplicadas. $\mathrm{O}$ fato do número de novo nó sem frutos variar inversamente com o número de nó com frutos ( $\mathrm{r}$ de Pearson -0,76), com o maior valor $(7,5)$ na suspensáo da irrigação por 70 dias, ajuda a explicar parte deste resultado.

No regime hídrico com maior período de suspensão da irrigação, $\mathrm{SI}_{109}$, o comprimento e a área foliar do ramo $\mathrm{r}_{2}$, proporcionaram as maiores médias $(49,8$ e 1078,7), diferindo significativamente dos outros dois regimes. Nestas duas variáveis de crescimento vegetativo as maiores doses de $\mathrm{P}_{2} \mathrm{O}_{5}$ proporcionaram os maiores valores, conforme esperado.

O número de novo nó sem fruto, comprimento e área foliar tiveram coeficiente de correlaçáo negativa muito forte $(-0,99$ a $-0,70)$ com o número de frutos, evidenciando que estas variáveis de crescimento vegetativo variam inversamente com o crescimento reprodutivo representado pelo número de frutos.

O número de nó com fruto, de frutos e de frutos por nó foram significativamente reduzidos $(4,9 ; 19,8$; e 2,9$)$ quando submetidos a 109 dias de suspensão da irrigação, mas não diferiram nos regimes hídricos $\mathrm{SI}_{70} \mathrm{e} \mathrm{I}$. O déficit hídrico prolongado ajuda a explicar a redução nestas variáveis de crescimento reprodutivo. O crescimento reprodutivo representado por estas três variáveis proporcionou equaçóes de regressão com os mais elevados coeficientes de determinação $(0,99 ; 1,00 ; 1,00)$.

O número de nó com frutos e de frutos por nó não diferiram significativamente em relação às doses de 100, 200 e $400 \mathrm{~kg} \mathrm{ha}^{-1}$ ano $^{-1} \mathrm{de}_{2} \mathrm{O}_{5}$, sugerindo que estas variáveis em ramos de $1^{\mathrm{a}}$ colheita podem ser menos responsivas às doses de fósforo utilizadas. Todavia, o número de frutos do ramo 
Tabela 4. Ramo plagiotrópico $\mathrm{r}_{2}$ com folhas e frutos em $1 .{ }^{\mathrm{a}}$ produção. Número de novo nó, comprimento $(\mathrm{cm})$, área foliar $\left(\mathrm{cm}^{2}\right)$; e número de nó com fruto, de frutos e de frutos por nó do ramo $\mathrm{r}_{2}$ obtido acima de 1,60 m do cafeeiro Rubi MG 1192, em maio/2008, em função de três regimes hídricos $(\mathrm{RH})$ : irrigação o ano todo (I); suspensão da irrigação por 70 dias $\left(\mathrm{SI}_{70}\right)$, a partir de 24 de junho; e por 109 dias $\left(\mathrm{SI}_{109}\right)$, quando a chuva induziu a floração; e de quatro doses de $\mathrm{P}_{2} \mathrm{O}_{5}: 0\left(\mathrm{P}_{0}\right), 100\left(\mathrm{P}_{100}\right), 200\left(\mathrm{P}_{200}\right)$ e $400\left(\mathrm{P}_{400}\right) \mathrm{kg} \mathrm{ha}^{-1}$ ano $\mathrm{g}^{-1}$; e respectivas equações de regressão, $y=f(x)$, sendo $x$ a dose de fósforo, $R^{2}$ e significância

\begin{tabular}{|c|c|c|c|c|c|c|c|c|}
\hline RH & $P_{0}$ & $\mathbf{P}_{100}$ & $\mathbf{P}_{200}$ & $\mathbf{P}_{400}$ & Méd. & $y=f(x)$ & $\mathbf{R}^{2}$ & \\
\hline \multicolumn{9}{|c|}{ Número de novo nó sem frutos } \\
\hline I & $13,7 \mathrm{ab}$ & $13,1 \mathrm{ab}$ & $14,7 \mathrm{ab}$ & $13,1 \mathrm{ab}$ & 13,6 & $y=-2 \cdot 10^{-5} x^{2}+0,0074 x+13,39$ & 0,31 & NS \\
\hline $\mathrm{SI}_{70}$ & $10,6 \mathrm{c}$ & $10,6 \mathrm{c}$ & $12,5 \mathrm{bc}$ & $14,9 a b$ & 12,1 & $y=0,0113 x+10,12$ & 0,94 & * \\
\hline $\mathrm{SI}_{109}$ & $14,5 \mathrm{ab}$ & $14,0 \mathrm{ab}$ & $13,8 \mathrm{ab}$ & $15,2 \mathrm{a}$ & 14,4 & $y=3.10^{-5} x^{2}-0,0084 x+14,525$ & 0,99 & NS \\
\hline Méd. & 12,9 & 12,6 & 13,7 & 14,4 & & & & \\
\hline \multicolumn{9}{|c|}{ Comprimento } \\
\hline I & 38,4 & 46,7 & 51 & 50,1 & $46,6 \mathrm{C}$ & $y=-0,0002 x^{2}+0,0977 x+38,47$ & 1,00 & * \\
\hline $\mathrm{SI}_{70}$ & 42,9 & 43,3 & 50,3 & 54,5 & $47,7 \mathrm{~B}$ & $y=0,0316 x+42,22$ & 0,92 & * \\
\hline $\mathrm{SI}_{109}$ & 44,7 & 46 & 53 & 55,5 & $49,8 \mathrm{~A}$ & $y=-4 \cdot 10^{-5} x^{2}+0,0456 x+43,92$ & 0,91 & NS \\
\hline Méd. & 41,2 b1 & $45,3 b$ & $51,4 \mathrm{a}$ & $53,4 \mathrm{a}$ & & $y=-8.10^{-5} x^{2}+0,0656 x+40,76$ & 0,97 & NS \\
\hline \multicolumn{9}{|c|}{ Área Foliar } \\
\hline I & 386 & 407 & 538 & 6719 & $501 \mathrm{~B}$ & $y=0,7578 x+367,96$ & 0,96 & * \\
\hline $\mathrm{SI}_{70}$ & 274 & 382 & 590 & 1115 & $590 \mathrm{~B}$ & $y=0,0028 x^{2}+0,9859 x+268,79$ & 1,00 & * \\
\hline $\mathrm{SI}_{109}$ & 859 & 897 & 1381 & 1177 & $1079 \mathrm{~A}$ & $y=-0,0062 x^{2}+3,5149 x+790,41$ & 0,69 & NS \\
\hline Méd. & $506 b^{1}$ & $562 b$ & $836 \mathrm{a}$ & $988 \mathrm{a}$ & & $y=1,285 x+498,28$ & 0,93 & * \\
\hline \multicolumn{9}{|c|}{ Número de nó com frutos } \\
\hline I & 5,7 & 7,1 & 6,8 & 8,7 & $7,1 \mathrm{~A}$ & $y=1.10^{-6} x^{2}+0,0063 x+5,91$ & 0,89 & NS \\
\hline $\mathrm{SI}_{70}$ & 7,0 & 6,6 & 8,9 & 7,1 & $7,5 \mathrm{~A}$ & $y=-3.10^{-5} x^{2}+0,0135 x+6,60$ & 0,39 & NS \\
\hline $\mathrm{SI}_{109}$ & 2,0 & 6,0 & 6,0 & 5,7 & $4,9 \mathrm{~B}$ & $y=-7.10^{-5} x^{2}+0,035 x+2,32$ & 0,89 & NS \\
\hline Méd. & $4,9 \mathrm{~b}$ & $6,5 \mathrm{a}$ & $7,2 \mathrm{a}$ & $7,3 \mathrm{a}$ & & $y=-3.10^{-5} x^{2}+0,0175 x+4,94$ & 0,99 & NS \\
\hline \multicolumn{9}{|c|}{ Número de frutos } \\
\hline I & 27,8 & 45,9 & 51,8 & 61,5 & $46,8 \mathrm{~A}$ & $y=-0,0002 x^{2}+0,1683 x+28,74$ & 0,98 & NS \\
\hline $\mathrm{SI}_{70}$ & 31,3 & 36,4 & 56,8 & 62,8 & $46,8 \mathrm{~A}$ & $y=-0,0002 x^{2}+0,1469 x+29,10$ & 0,92 & NS \\
\hline $\mathrm{SI}_{109}$ & 3,7 & 19,7 & 22,8 & 33 & $19,8 \mathrm{~B}$ & $y=-0,0002 x^{2}+0,1333 x+4,864$ & 0,92 & NS \\
\hline Méd. & $22,6 \mathrm{c}$ & $34,3 \mathrm{bc}$ & $41,8 \mathrm{ab}$ & $52,4 \mathrm{a}$ & & $y=-0,0001 x^{2}+0,1205 x+22,82$ & 1,00 & * \\
\hline \multicolumn{9}{|c|}{ Número de frutos/nó } \\
\hline I & 3,8 & 7,2 & 7,4 & 7,9 & $6,5 \mathrm{~A}$ & $y=-5.10^{-5} x^{2}+0,0293 x+4,064$ & 0,92 & NS \\
\hline $\mathrm{SI}_{70}$ & 4,4 & 4,5 & 7 & 8,8 & $6,2 \mathrm{~A}$ & $y=0,0112 x+4,100$ & 0,93 & * \\
\hline $\mathrm{SI}_{109}$ & 1,6 & 2,6 & 3,5 & 4 & $2,9 \mathrm{~B}$ & $y=-2.10^{-5} x^{2}+0,0126 x+1,573$ & 0,99 & * \\
\hline Méd. & $3,3 \mathrm{~b}$ & $4,7 \mathrm{ab}$ & $5,9 \mathrm{a}$ & $6,9 \mathrm{a}$ & & $y=-2.10^{-5} x^{2}+0,0167 x+3,278$ & 1,00 & * \\
\hline
\end{tabular}

Médias seguidas pela mesma letra minúscula na linha e maiúscula na coluna não diferem entre si ao nível de probabilidade de 5\%.

*: Significativo a $5 \%$ probabilidade. NS: náo significativo a $5 \%$ de probabilidade.

$\mathrm{r}_{2}$, também foi influenciado significativamente pelas doses de fósforo com maiores valores $(41,8$ e 52,4) nas doses de 200 e 400 que não diferiram significativamente entre si.

Assim, o crescimento reprodutivo representado pelo maior número de frutos do ramo $r_{2}$ reduziu sistematicamente a distribuiçáo de fotoassimilados para as estruturas vegetativas representadas pelo comprimento e área foliar do ramo. O que está de acordo com Amaral et al. (2006) ao relatarem que os frutos são fortes drenos, limitam a mobilização de assimilados para outros órgãos da planta e reduzem o crescimento vegetativo. DAмatTA et al. (2008) também mostraram que nas plantas em que os frutos foram removidos, o comprimento e a área foliar foram maiores do que nas plantas sem remoção dos frutos.

\section{Ramos plagiotrópicos tipo $r_{3}$}

Este é o ramo de $2^{\text {a }}$ colheita. Provavelmente, tenha surgido após o retorno da irrigação em setembro/2005, proporcionando as safras de 2006/2007 e 2007/2008, quando foi analisado. Neste ramo, o número de nó com frutos foi afetado por dupla interação entre regime hídrico e dose de fósforo (Tabela 5). Já o número de novo nó sem frutos e de 
Tabela 5. Ramo plagiotrópico $\mathrm{r}_{3}$ com ramificação secundária e outras e frutos em $2 .^{\mathrm{a}}$ produção. Número de novo nó sem frutos e de ramificação secundária; comprimento $(\mathrm{cm})$, área foliar $\left(\mathrm{cm}^{2}\right)$; e número de nó com frutos e de frutos do ramo $\mathrm{r}_{3}$ obtido acima de 1,60 $\mathrm{m}$ do cafeeiro Rubi MG 1192, em maio/2008, em função de três regimes hídricos (RH): irrigação o ano todo (I); suspensão da irrigação a partir de 24 de junho por 70 dias $\left(\mathrm{SI}_{70}\right)$, até a chuva induzir a floração, aos 109 dias $\left(\mathrm{SI}_{100}\right)$; e de quatro doses de adubação fosfatada: 0 ( $\left.\mathrm{P}_{0}\right)$, 100 $\left(\mathrm{P}_{100}\right), 200\left(\mathrm{P}_{200}\right)$ e $400\left(\mathrm{P}_{400}\right)$ kg de $\mathrm{P}_{2} \mathrm{O}_{5}$ ha $^{-1}$; e respectivas equaçóes de regressão, $\mathrm{y}=\mathrm{f}(\mathrm{x})$, sendo $\mathrm{x}$ a dose de fósforo, $\mathrm{R}^{2}$ e significância

\begin{tabular}{|c|c|c|c|c|c|c|c|c|}
\hline RH & $P_{0}$ & $\mathbf{P}_{100}$ & $\mathbf{P}_{200}$ & $\begin{array}{c}\mathbf{P}_{400} \\
\text { Número de } \mathrm{n}\end{array}$ & $\begin{array}{l}\text { Méd. } \\
\text { Jvo nó ser }\end{array}$ & $y=f(x)$ & $\mathbf{R}^{2}$ & \\
\hline I & 9,6 & 10,9 & 11,3 & 11,9 & $10,9 \mathrm{~B}$ & $y=-2 \cdot 10^{-5} x^{2}+0,012 x+9,67$ & 0,98 & NS \\
\hline $\mathrm{SI}_{70}$ & 8,3 & 9,4 & 11,4 & 12,8 & $10,5 \mathrm{~B}$ & $y=0,0115 x+8,46$ & 0,98 & * \\
\hline $\mathrm{SI}_{109}$ & 13,5 & 12,3 & 14,7 & 20,5 & $15,3 \mathrm{~A}$ & $y=7.10^{-5} x^{2}-0,0091 x+13,23$ & 0,98 & NS \\
\hline Méd. & $10,5 b^{1}$ & $10,9 \mathrm{~b}$ & $12,5 \mathrm{ab}$ & $15,0 \mathrm{a}$ & & $y=1.10^{-5} x^{2}+0,0072 x+10,38$ & 0,99 & * \\
\hline \multicolumn{9}{|c|}{ Número de ramificação secundária } \\
\hline I & 11,2 & 11,2 & 11,6 & 13,7 & $11,9 \mathrm{~B}$ & $y=2.10^{-5} x^{2}-0,0022 x+11,20$ & 1,00 & ** \\
\hline $\mathrm{SI}_{70}$ & 13,6 & 16,1 & 21,6 & 19,1 & $17,6 \mathrm{~A}$ & $y=-0,000 x^{2}+0,058 x+13,0$ & 0,87 & NS \\
\hline $\mathrm{SI}_{109}$ & 16,0 & 15,5 & 20,8 & 24,7 & $19,2 \mathrm{~A}$ & $y=2 \cdot 10^{-5} x^{2}+0,0175 x+15,34$ & 0,91 & * \\
\hline Méd. & $13,6 \mathrm{~b}$ & $14,3 b$ & $18,1 \mathrm{a}$ & $19,1 \mathrm{a}$ & & $y=-3.10^{-5} x^{2}+0,025 x+13,17$ & 0,90 & NS \\
\hline \multicolumn{9}{|c|}{ Comprimento } \\
\hline I & 81,6 & 85,3 & 92,6 & 99,2 & $89,7 \mathrm{~A}$ & $y=0,0452 x+81,76$ & 0,98 & * \\
\hline $\mathrm{SI}_{70}$ & 88,1 & 84,8 & 88,7 & 96,9 & $89,6 \mathrm{~A}$ & $y=0,0001 x^{2}-0,024 x+87,52$ & 0,95 & NS \\
\hline $\mathrm{SI}_{109}$ & 79,7 & 85,8 & 95,3 & 98,7 & $89,9 \mathrm{~A}$ & $y=-0,0001 x^{2}+0,099 x+79,0$ & 0,97 & NS \\
\hline Méd. & $83,1 \mathrm{c}$ & $85,3 c$ & $92,2 b$ & $98,3 \mathrm{a}$ & & $y=0,0398 x+82,76$ & 0,97 & * \\
\hline \multicolumn{9}{|c|}{ Área Foliar } \\
\hline I & 2440 & 4134 & 5436 & 4307 & $4079 \mathrm{~A}$ & $y=-0,0487 x^{2}+24,36 x+2370$ & 0,99 & NS \\
\hline $\mathrm{SI}_{70}$ & 3607 & 3306 & 4530 & 5084 & $4132 \mathrm{~A}$ & $y=0,0027 x^{2}+3,191 x+3430$ & 0,81 & NS \\
\hline $\mathrm{SI}_{109}$ & 3935 & 4236 & 5144 & 6223 & $4885 \mathrm{~A}$ & $y=5,9703 x+3840$ & 0,98 & ** \\
\hline Méd. & $3327 \mathrm{~b}$ & $3892 a b$ & 5037 a & $5205 a$ & & $y=-0,0149 x^{2}+10,998 x+3222$ & 0,94 & NS \\
\hline \multicolumn{9}{|c|}{ Número de nó com frutos } \\
\hline I & 4,3 def & $5,1 \mathrm{de}$ & 7,4 bcd & $12,6 \mathrm{a}$ & 7,3 & $y=0,0215 x+3,58$ & 0,97 & * \\
\hline $\mathrm{SI}_{70}$ & 5,4 de & 5,9 cde & $9,5 a b$ & $10,3 \mathrm{abc}$ & 7,7 & $y=-2 \cdot 10^{-5} x^{2}+0,023 x+4,97$ & 0,88 & NS \\
\hline $\mathrm{SI}_{109}$ & $1,0 \mathrm{f}$ & 2,3 ef & 3,0 ef & 3,0 ef & 2,3 & $y=-3.10^{-5} x^{2}+0,015 x+1,01$ & 1,00 & * \\
\hline Méd. & 4,3 & 5,1 & 7,4 & 12,6 & 7,3 & & & \\
\hline \multicolumn{9}{|c|}{ Número de frutos } \\
\hline I & 63 & 88 & 179 & 182 & $136 \mathrm{~A}$ & $y=-0,001 x^{2}+0,722 x+53,01$ & 0,88 & NS \\
\hline $\mathrm{SI}_{70}$ & 121 & 130 & 195 & 199 & $153 \mathrm{~A}$ & $y=-0,0005 x^{2}+0,441 x+112,9$ & 0,85 & NS \\
\hline $\mathrm{SI}_{109}$ & 12 & 15 & 46 & 60 & $33 \mathrm{~B}$ & $y=0,1314 x+10,10$ & 0,90 & * \\
\hline Méd. & $68 \mathrm{~b}$ & $74 \mathrm{~b}$ & $141 \mathrm{a}$ & $146 \mathrm{a}$ & & $y=-0,0005 x^{2}+0,418 x+59,96$ & 0,83 & NS \\
\hline
\end{tabular}

Médias seguidas pela mesma letra minúscula na linha e maiúscula na coluna não diferem entre si ao nível de probabilidade de $5 \%$.

*; **: Significativo a 5\% Significativo e $1 \%$ de probabilidade respectivamente. NS: náo significativo a 5\% de probabilidade.

ramificação secundária, comprimento e área foliar, e número de nó com frutos e de frutos do ramo foram afetados diretamente pelas doses de $\mathrm{P}_{2} \mathrm{O}_{5}$, o que era esperado pelo aumento deste nutriente na solução do solo e validado em conjunto ou ratificado pelas equaçōes de regressóes ajustadas com elevados coeficientes de determinação $(>0,83)$.

$\mathrm{O}$ aparecimento de novos nós sem frutos $(10,9$ e 10,5) não diferiu entre os tratamentos $\mathrm{I}_{\text {e }} \mathrm{SI}_{70}$, mostrando comportamento diferenciado em relação ao ramo $r_{2}$. Por outro lado, a suspensão da irrigação por 109 dias aumentou significativamente $(15,3)$ esta variável. Tal aumento parece estar associado à menor presença de frutos, sugerindo maior crescimento vegetativo na extremidade do ramo em $2^{a}$ produção, em detrimento do crescimento reprodutivo, o que é respaldado pela correlação negativa muito forte $(-0,77)$ entre novos nós e número de frutos.

O número de ramificação secundária foi reduzido $(11,9)$ significativamente na irrigação o ano todo e nas do- sagens de 0 e $100 \mathrm{~kg}$ de $\mathrm{P}_{2} \mathrm{O}_{5}$. Por outro lado, foi aumentado $(17,6$ e 19,2) quando houve suspensão da irrigação, respectivamente, por 70 e 109 dias e nas dosagens de 200 e $400 \mathrm{~kg}$ de $\mathrm{P}_{2} \mathrm{O}_{5}$. No regime hídrico $\mathrm{SI}_{70}$, o maior número de ramificaçáo secundária $(17,6)$ proporcionou o maior número de frutos $(152,9)$, o que é esperado em razão do aumento de ramos produtivos. Todavia, no regime hídrico $\mathrm{SI}_{109}$, o maior número de ramificação secundária $(19,20)$, proporcionou o menor número de frutos $(33,13)$. O fato de o número de ramificação secundária não diferir nos tratamentos com suspensão da irrigação e proporcionar diferentes número de frutos vai de encontro as duas hipóteses que: um déficit hídrico excessivo $\left(\mathrm{SI}_{109}\right)$ pode proporcionar a redução do número de frutos e maior crescimento vegetativo; um déficit hídrico adequado $\left(\mathrm{SI}_{70}\right)$ pode proporcionar o aumento do número de frutos.

$\mathrm{O}$ comprimento do ramo e a área foliar não foram afetados pelo regime hídrico, mas sim pelas doses de $\mathrm{P}_{2} \mathrm{O}_{5}$. 
Em termos de regime hídrico, estes resultados diferem dos observados para os ramos $r_{1}$ e $r_{2}$, cujos valores aumentaram com a suspensão da irrigação. Tal fato sugere que a suspensão da irrigaçáo durante o período de reduzido crescimento da planta tem maior influência em um ramo de $1 .{ }^{\mathrm{a}}$, em comparaçáo com um de $2 .{ }^{\mathrm{a}}$ produção.

Convém realçar a variação semelhante de todas as variáveis do ramo $\mathrm{r}_{3}$ de aumento quadrático com o aumento das doses de fósforo, validado por coeficientes de determinação elevados $(>0,83)$. A dose de $400 \mathrm{~kg}$ de $\mathrm{P}_{2} \mathrm{O}_{5}$ $\mathrm{ha}^{-1}$ proporcionou as maiores médias para os crescimentos vegetativo e reprodutivo analisados.

Mesmo havendo mobilização de nutrientes pelos frutos da safra em formação, no período de crescimento reprodutivo, a planta mantém o crescimento vegetativo, particionando os fotoassimilados de forma diferenciada, segundo o maior ou menor desenvolvimento reprodutivo. Essa partição de fotoassimilados pode estar relacionada com o suprimento de água, fertilizante ou outro fator ambiental, assim, em tese, é possível reduzir a bienalidade da produção do cafeeiro. De acordo com Laviola et al. (2007), as práticas culturais podem maximizar a produção de fotoassimilados nos períodos mais críticos, de forma
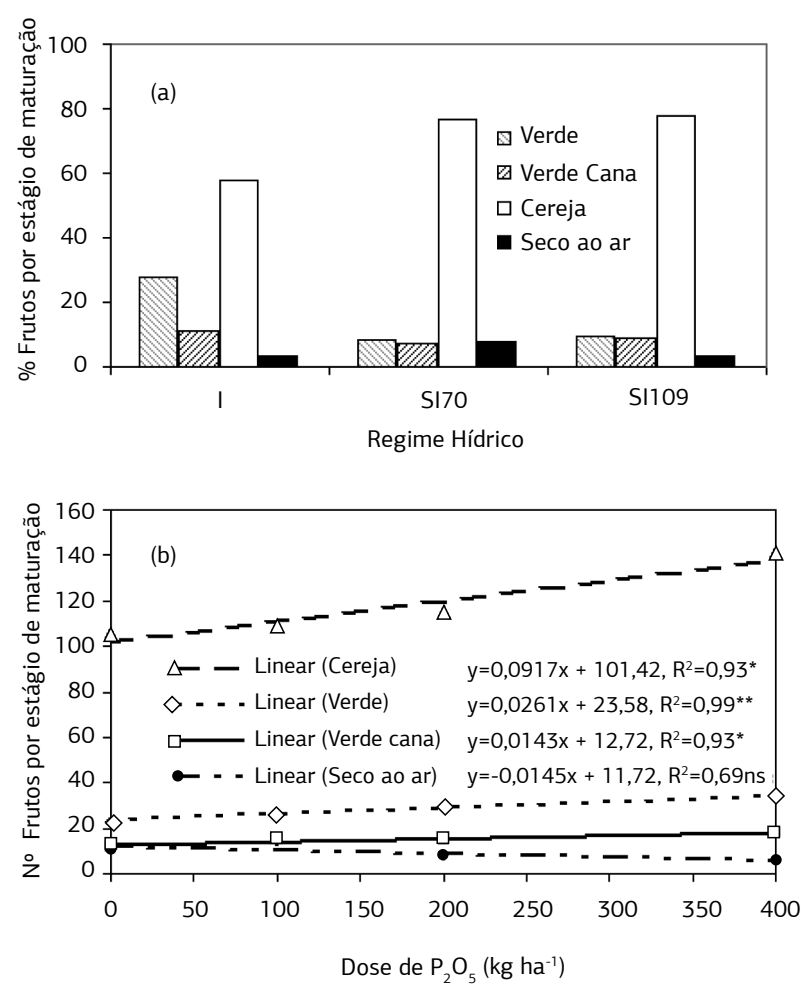

Figura 2. Porcentagem e número de frutos por estágio de maturaçáo: verde, verde cana, cereja e seco ao ar obtido acima de 1,60 m do cafeeiro Rubi MG 1192, em maio/2008, em função de: (a) de três regimes hídricos: sem suspensão da irrigação, feita o ano todo (I); e a partir de 24/6/07, com suspensáo da irrigaçáo por 70 dias $\left(\mathrm{SI}_{70}\right)$, até $02 / 09$; com suspensão da irrigação por 109 dias $\left(\mathrm{SI}_{100}\right)$, até $11 / 10$, quando a chuva de $12 \mathrm{~mm}(01 / 10)$ e as temperaturas mínimas mais elevadas, visualmente, induziram a floração; (b) de quatro doses de $\mathrm{P}_{2} \mathrm{O}_{5}:$ 0, 100, 200 e $400 \mathrm{~kg} \mathrm{ha}^{-1}$. que a planta venha a produzir carboidratos em quantidades suficientes para o desenvolvimento dos frutos e para manutenção do crescimento vegetativo, reduzindo a bienalidade de produção.

Entretanto, a demanda por nutrientes não varia somente em função da produção, pois quando esta é baixa os ramos plagiotrópicos, a formação e expansão de folhas e de novos ramos constituem o dreno de carboidratos e nutrientes (Malavolta et al., 2002). Este pode ter sido um dos fatores para que o tratamento $\mathrm{SI}_{109}$ proporcionasse maior crescimento vegetativo, tanto para o ramo ortotrópico como para os ramos plagiotrópicos e menor crescimento reprodutivo. Além disso, sob condições limitantes de água ou nutrientes, o maior crescimento reprodutivo da safra em formação, pode implicar menor crescimento vegetativo uma vez que estas duas fases ocorrem simultaneamente no cafeeiro adulto.

\section{Número de frutos em função do estágio de maturação}

Não houve interação entre os regimes hídricos e a adubação fosfatada. O número de frutos em cada estágio de maturação foi afetado pelo regime hídrico, mas não pelas doses de $\mathrm{P}_{2} \mathrm{O}_{5}$ (Figura 2b). O percentual de frutos por estágio de maturação náo diferiu significativamente entre os tratamentos com irrigação o ano todo e com suspensão da irrigação por 70 dias (Figura 2a).

$\mathrm{O}$ tratamento com irrigação o ano todo proporcionou os menores porcentuais de frutos cereja $(57,9 \%)$ e menoresde frutos verdes $(27,7 \%)$, provavelmente decorrente da menor uniformidade da floração. Por outro lado, os tratamentos com suspensão da irrigaçáo por 70 e 109 dias proporcionaram os maiores porcentuais de frutos cereja $(76,9$ e $77,9 \%)$ e menores de frutos verdes $(8,3$ e $9,6 \%)$, respectivamente, sugerindo ter havido maior uniformidade da floração em ambos os tratamentos. Quando comparada à suspensão da irrigação por 70 dias, a irrigaçáo o ano todo proporcionou menos 19\% de frutos cereja e $19,4 \%$ a mais de frutos verdes.

Estes resultados evidenciam que a suspensão da irrigação por determinado período anterior a fase de floração do cafeeiro, pode induzir uma floração mais uniforme após o retorno da irrigação ou chuva, e consequentemente maior uniformidade de maturação dos frutos, aumentando a porcentagem de frutos cereja e reduzindo a porcentagem de frutos nos outros estágios de maturação no momento da colheita.

A suspensão da irrigação por 109 dias resultou em porcentual de maturação dos frutos semelhante à suspensão por 70 dias. No entanto, o prolongamento do déficit hídrico pode ter sido um dos fatores para esse tratamento ter o menor número total de frutos dos ramos $\mathrm{r}_{2}$ e $\mathrm{r}_{3}$.

Embora o número de frutos por estágio de maturação não tenha diferido significativamente entre as doses 
de $\mathrm{P}_{2} \mathrm{O}_{5}$, ocorreu o esperado aumento do número total de frutos cereja, verde cana e verde com a consequente redução de frutos secos ao ar em funçáo do aumento das doses de $\mathrm{P}_{2} \mathrm{O}_{5}$ (Figura 2b).

\section{Produtividade em sacas de $\mathbf{6 0} \mathbf{~ k g}$ de grãos de café beneficiado ha-1}

Houve interação entre regime hídrico e adubação fosfatada (Figura 3) em relação à produtividade. A dupla interação também observada no número de novo nó sem frutos e de folhas do ramo $r_{1}$ (crescimento vegetativo); no número de novo nó do ramo $\mathrm{r}_{2}$ (crescimento vegetativo) e de nó com frutos do ramo $\mathrm{r}_{3}$ (crescimento reprodutivo) que também interagiram, ajudam a explicar este resultado.

Muito embora o modelo linear pudesse ser utilizado nos intervalos estudados, nos três regimes hídricos foi utilizado o modelo quadrático por ter o melhor coeficiente de determinaçáo, que possibilita melhores previsóes, e representar melhor as relaçóes causa-efeito esperadas, no intervalo utilizado, entre as doses crescentes de fósforo e a produtividade do cafeeiro.

No intervalo de 0 a $400 \mathrm{~kg}$ de $\mathrm{P}_{2} \mathrm{O}_{5}$ ha $^{-1}$, os tratamentos: $\mathrm{I}_{1} \mathrm{SI}_{70}$ e $\mathrm{SI}_{109}$, atingiram o ponto máximo com as aplicaçôes de 250,5, 400 e 202,5 kg ha' ${ }^{-1}$ de $\mathrm{P}_{2} \mathrm{O}_{5}$ respectivamente. Porém, o tratamento com suspensáo da irrigaçáo por 70 dias não chegou a expressar seu potencial máximo de produtividade, sugerindo, sem fazer extrapolaçóes, ser possível a utilização de doses maiores de $\mathrm{P}_{2} \mathrm{O}_{5}$, não necessariamente mais econômicas, para se obter produtividades maiores.

As menores taxas de aumento da produtividade obtidas com o aumento das doses de $\mathrm{P}_{2} \mathrm{O}_{5}$, pelo modelo ajustado para o tratamento com irrigaçáo o ano todo, em comparação com a suspensão da irrigação por 70 dias, além de poder estar relacionadas com variáveis de crescimento vegetativo que interagiram, também podem estar relacionadas com o menor pegamento de diversas floradas. Todavia, os dados de crescimento reprodutivo em termos de número de nó com frutos, número de frutos e número de frutos/nó não diferiram entre os regimes hídricos $\mathrm{I}_{\text {e }} \mathrm{SI}_{70}$.

No tratamento com suspensão da irrigação por 70 dias, todas as doses de $\mathrm{P}_{2} \mathrm{O}_{5}$ ha $^{-1}$ proporcionaram produtividades mais elevadas que os demais regimes hídricos. Dentre outros fatores, as elevadas produtividades observadas podem estar relacionadas com a suspensão da irrigaçáo por um período adequado e retorno da irrigação quando as temperaturas máximas estavam propícias para a floração uniforme, que proporcionou a maior porcentagem de frutos cereja, e com o maior número de frutos observados nos ramos $r_{2}$ e $r_{3}$.

Por outro lado, a suspensão da irrigação por 109 dias causou reflexos negativos na produtividade e está de acordo com a hipótese de um déficit hídrico excessivo, que levou à redução no número de nó com frutos e no núme-

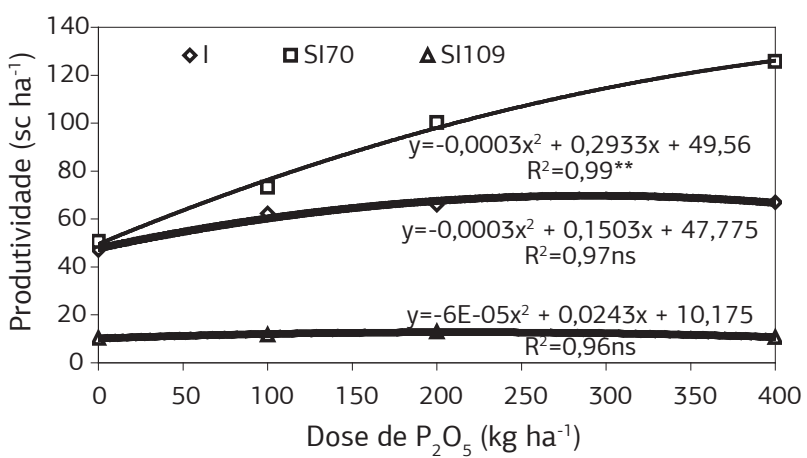

Figura 3. Produtividade média em sacas de $60 \mathrm{~kg}$ de grãos de café beneficiado ha ${ }^{-1}$ da safra 2007/2008 do cafeeiro Rubi MG 1192, em função de quatro doses de $\mathrm{P}_{2} \mathrm{O}_{5}: 0,100,200$ e $400 \mathrm{~kg} \mathrm{ha}^{-1}$ e de três regimes hídricos: sem suspensão da irrigação, feita o ano todo (I); e a partir de 24/6/07, com suspensão da irrigação por 70 dias $\left(\mathrm{SI}_{70}\right)$, até 02/09; com suspensão da irrigaçáo por 109 dias $\left(\mathrm{SI}_{109}\right)$, até $11 / 10$, quando a chuva de $12 \mathrm{~mm}(01 / 10)$ e as temperaturas mínimas mais elevadas, visualmente, induziram a floração.

ro de frutos observados nos ramos $r_{2}$ e $r_{3}$. Muito embora a floração possa ter sido intensa e uniforme, pode ter sido prejudicada pelas altas temperaturas médias de $32^{\circ} \mathrm{C}$ no momento da floração, entre 11/10 e 16/10, entretanto, maiores consideraçôes não podem ser feitas, uma vez que as respectivas florações não foram avaliadas e em razão dos dados dos regimes hídricos I e SI70 não diferirem estatisticamente em termos de número de frutos.

Convém ressaltar que as plantas do cafeeiro estavam no segundo ano de produção após o decote e esqueletamento, seguindo padróes próprios de crescimento e desenvolvimento em função da arquitetura da planta já estabelecida acima e abaixo da superfície do solo.

Esses resultados permitem afirmar que cafeeiros em fase reprodutiva/vegetativa respondem à adubação fosfatada tanto em crescimento vegetativo e reprodutivo quanto em produtividade, dependendo do manejo da irrigação no período seco do ano, quando os dias são mais frios e curtos e antecedem à floraçáo.

\section{CONCLUSÃO}

Os regimes hídricos e as doses de $\mathrm{P}_{2} \mathrm{O}_{5}$ interagem e afetam algumas variáveis agronômicas de crescimento do cafeeiro. A altura da planta, o número de nó e de ramos primários do ramo ortotrópico e o número de frutos por estágio de maturação não são afetados pelas doses de $\mathrm{P}_{2} \mathrm{O}_{5}$. $\mathrm{O}$ comprimento e a área foliar do ramo; o número de frutos e de frutos por nó por ramo reprodutivo aumenta com a dose de $\mathrm{P}_{2} \mathrm{O}_{5}$. Os regimes hídricos de suspensão da irrigação por 70 e 109 dias após a colheita e anterior ao florescimento proporcionam as maiores porcentagens de frutos cereja; e o respectivo maior e menor número de frutos por ramo e de produtividade de grãos. $\mathrm{O}$ regime hídrico sem 
suspensão da irrigação proporciona menor porcentagem de frutos cereja em relação aos tratamentos com suspensão da irrigação, menor ramo $r_{1}$ e menor produtividade de grãos em relação à suspensão da irrigação por 70 dias.

\section{REFERÊNCIAS}

AMARAL, J.A.T.; RENA, A.B.; AMARAL, F.A.T. Crescimento vegetativo sazonal do cafeeiro e sua relação com fotoperíodo, frutificação, resistência estomática e fotossíntese. Pesquisa Agropecuária Brasileira, v.41, p.377-384, 2006.

CAMARGO, A.P.; CAMARGO, M.B.P. Definição e esquematização das fases fenológicas do cafeeiro arábica nas condiçóes tropicais do Brasil. Bragantia, v.60, p.65-68, 2001.

CARR, M.K.V. The water relations and irrigation requirements of coffee. Experimental Agriculture, v.37, p.1-36, 2001.

CONAB: Acompanhamento da safra brasileira. Café, 2009. Disponível em: http://www.conab.gov.br/conabweb/download/ safra/4cafe08.pdf. Acesso em: 26 jan. 2009.

CRISOTO, C.H.; GRANTZ, D.A.; MENZEIR, F.C. Effects of water deficit on flower opening in coffee (Coffea arabica L). Tree Physiology, v.10, p.127-139, 1992.

DAMATTA, F. M.; CUNHA, R. L.; ANTUNES, W. C.; MARTINS, S. C. V.; ARAUJO, W. L.; FERNIE, A.R.; MORAES, G.A.B.K. In field-grown coffee trees source sink manipulation alters photosynthetic rates, independently of carbon metabolism, via alterations in stomatal function. New Phytologist, v.178, p.348$357,2008$.

DAMATTA, F.M.; RENA, A.B. Relações hídricas no cafeeiro. In: SIMPÓSIO DE PESQUISA DOS CAFÉS DO BRASIL, 1., 2002, Brasília, DF. Palestras... Brasília, DF: Embrapa Café, 2002. p.9-44.

DAMATTA, F.M.; RONCHI, C.P.; MAESTRI, M.; BARROS, R.S. Ecophysiology of coffee growth and production. Brazilian Journal of Plant Physiology, v.19, p.485-510, 2007.

DRINNAN, J.E.; MENZEL, C.M. Synchronization of anthesis and enhancement of Vegetative growth in coffee (Coffea arabica L.) following water-stress during flower initiation. Journal of Horticultural Science, v.69, p.841-849, 1994.
EMBRAPA. Centro Nacional de Pesquisa de Solos. Manual de métodos de análise de solo. 2.ed. Rio de Janeiro, 1997. 212p.

GUERRA, A.F.; ROCHA, O.C.; RODRIGUES, G.C.; SANZONOWICZ, C. Manejo da irrigação do cafeeiro, com estresse hídrico controlado, para uniformização de florada. In: ZAMBOLIM, L. (Ed.). Boas práticas agrícolas na produçấo de café. Viçosa, 2006. Cap. 3, p.83-115.

GUERRA, A.F.; ROCHA, O.C.; RODRIGUES, G.C.; SANZONOWICZ, C.; FILHO, G.C.R.; TOLEDO, P.M.R.; RIBEIRO, L.F. Sistema de produção de café irrigado: um novo enfoque. Revista trimestral da Associação Brasileira de Irrigação e Drenagem - ABID, n.73, p.52-61, 2007.

LAVIOLA, B.G.; MARTINEZ, H.E.P.; SOUZA, R.B.; ALVAREZ VENEGAS, V.H. Dinâmica de cálcio e magnésio em folhas e frutos de cafeeiro arábico em três níveis de adubação. Revista Brasileira de Ciência do Solo, v.31, p.319-329, 2007.

MALAVOLTA, E.; FAVARIN, J.L.; MALAVOLTA, M.; CABRAL, C.P.; HEINRICHS, R.; SILVEIRA, J.S.M. Repartição de nutrientes nos ramos, folhas e flores do cafeeiro. Pesquisa Agropecuária Brasileira, v.37, p.1017-1022, 2002.

MATIELLO, J.B.; SANTINATO, R.; GARCIA, A.W.R.; ALMEIDA, S.R.; FERNANDES, D.R. Cultura de café no Brasil: novo manual de recomendaçóes. Rio de Janeiro: MAPA/ PROCAFÉ, 2005. 438 p.

NAZARENO, R.B.; OLIVEIRA, C.A.S.; SANZONOWICZ, C.; SAMPAIO, J.B.R.; SILVA, J.C.P.; GUERRA, A.F. Crescimento inicial do cafeeiro Rubi em resposta a doses de nitrogênio, fósforo e potássio e a regimes hídricos. Pesquisa Agropecuária Brasileira, v.38, p.903-910, 2003.

NASCIMENTO, L.M. Paralisação da irrigação e sincronia do desenvolvimento das gemas reprodutivas de cafeeiros orgânico e adensado. 2008. 72p. Dissertação (Mestrado em Agronomia) Faculdade de Agronomia e Medicina Veterinária, Universidade de Brasília, Distrito Federal.

SILVA, E.A.; BRUNINI, O.; SAKAI, E.; ARRUDA, F.B.; ARRUDA, F.B.; PIRES, R.C. M.P. Influência de déficits hídricos controlados na uniformização do florescimento e produção do cafeeiro em três diferentes condiçôes edafoclimáticas do Estado de São Paulo. Bragantia, v.68, p.493-501, 2009. 